\title{
Using Machine-Learning for Prediction of the Response to Cardiac Resynchronization Therapy: the SMART-AV Study
}

\author{
Stacey Howell ${ }^{1}$, Tim Stivland ${ }^{2}$, Kenneth Stein ${ }^{2}$, Kenneth Ellenbogen ${ }^{3}$, and Larisa \\ Tereshchenko ${ }^{1}$ \\ ${ }^{1}$ Oregon Health \& Science University School of Medicine \\ ${ }^{2}$ Boston Scientific Corp \\ ${ }^{3}$ Virginia Commonwealth University
}

December 1, 2020

\begin{abstract}
Introduction-We aimed to apply machine learning (ML) to develop a prediction model for cardiac resynchronization therapy (CRT) response. Methods and Results-Participants from the SmartDelay Determined AV Optimization (SMART-AV) trial ( $\mathrm{n}=741$; age, $66 \pm 11$ yrs; $33 \%$ female; 100\% NYHA III-IV; 100\% EF[?]35\%) were randomly split into training \& testing (80\%; $\mathrm{n}=593)$, and validation (20\%; $\mathrm{n}=148)$ samples. Baseline clinical, ECG, echocardiographic and biomarker characteristics, and left ventricular (LV) lead position (43 variables) were included in $6 \mathrm{ML}$ models (random forests, convolutional neural network, lasso, adaptive lasso, plugin lasso, elastic net, ridge, and logistic regression). A composite of freedom from death and heart failure hospitalization and a $>15 \%$ reduction in LV end-systolic volume index at 6-months post-CRT was the endpoint. The primary endpoint was met by 337 patients (45.5\%). The adaptive lasso model was more accurate than class I ACC/AHA guidelines criteria (AUC 0.759; 95\%CI 0.678-0.840 versus 0.639; 95\%CI 0.554-0.722; P<0.0001), well-calibrated, and parsimonious (19 predictors; nearly half are potentially modifiable). The model predicted CRT response with $70 \%$ accuracy, $70 \%$ sensitivity, and $70 \%$ specificity, and should be further validated in prospective studies. Conclusions - ML predicts short-term CRT response and thus may help with CRT procedure planning.
\end{abstract}

\section{Hosted file}

Smart AV ML 11_29_20.pdf available at https://authorea.com/users/380245/articles/496311using-machine-learning-for-prediction-of-the-response-to-cardiac-resynchronizationtherapy-the-smart-av-study 